\title{
A Modified Bairstow Method for Multiple Zeros of a Polynomial
}

\author{
By F. M. Carrano
}

\begin{abstract}
A modification of Bairstow's method to find multiple quadratic factors of a polynomial is presented. The nonlinear system of equations of the Bairstow method is replaced by high order partial derivatives of that system. The partials are computed by a repetition of the Bairstow recursion formulas. Numerical results demonstrate that the modified method converges in many cases where the Bairstow method fails due to the multiplicity of the quadratic factor. Rall [4] has described a generalization of Newton's method for simultaneous nonlinear equations with multiple roots. This may be applied to solve the nonlinear Bairstow equations; however, it fails in some cases due to near-zero divisors. Examples are presented which illustrate the behavior of the author's algorithm as well as the methods of Rall and Bairstow.
\end{abstract}

1. Introduction. Bairstow's method [1] is a well-known algorithm to determine quadratic factors of a polynomial with real coefficients. It is limited, however, in that convergence is quadratic only if the zeros are complex conjugate pairs of multiplicity one, or are real of multiplicity at most two. For higher multiplicities it is impractically slow or subject to failure. An extension of the Bairstow method is described which relaxes this limitation. An algorithm due to Rall [4] for solving simultaneous nonlinear equations with multiple roots is also discussed in the context of Bairstow's procedure.

2. Newton's Method. The ideas to be presented are analogous to Newton's method and some of its modifications, and hence we begin our discussion at this point.

Consider the polynomial $P(x)$ with zero $\alpha$. The Newton iteration function is

$$
x-P(x) / P^{\prime}(x) \text {. }
$$

If $\alpha$ is a zero of multiplicity $m$, we consider two modified iteration functions, both of which are quadratically convergent. The first is obtained by observing that $\alpha$ is a simple zero of $P^{(m-1)}(x)$. An application of Newton's method then gives

$$
x-P^{(m-1)}(x) / P^{(m)}(x) .
$$

The second (see e.g. [3]) is

$$
x-m P(x) / P^{\prime}(x) .
$$

If Horner's scheme is used to evaluate $P$ and its derivatives, (2) costs more than (3) to compute. To determine $m$ in (3), however, it may be necessary to calculate the derivatives used in (2) anyway. If such is the case, cost is no longer a factor, and the

Received August 6, 1969, revised December 26, 1972.

AMS (MOS) subject classifications (1970). Primary 65H05, 68A10, 12D10, 26-04, 26A78, 30-04, 30A08; Secondary 12D05.

Key words and phrases. Multiple zeros of a polynomial, root finding, Bairstow method, multiple quadratic factors of a polynomial. 
choice of method would depend upon potential numerical difficulties. In fact, such difficulties are likely as the denominator of (3) becomes small. Similar results will be encountered in the subsequent presentation. (1), (2), and (3) are analogous to the Bairstow, the modified Bairstow and the Rall methods, respectively, which are considered next.

3. Bairstow's Method. Consider the polynomial with real coefficients $P_{n}(x)=$ $a_{0} x^{n}+a_{1} x^{n-1}+\cdots+a_{n}$, and an approximation, $x^{2}-p x-q$, to a quadratic factor of $P_{n}(x)$. Form

$$
P_{n}(x)=\left(x^{2}-p x-q\right)\left(b_{0} x^{n-2}+b_{1} x^{n-3}+\cdots+b_{n-2}\right)+(x-p) b_{n-1}+b_{n},
$$

where $b_{i}=a_{i}+p b_{j-1}+q b_{i-2}, j=0, \cdots, n ; b_{-2}=b_{-1}=0$. Then $x^{2}-p x-q$ is a quadratic factor of $P_{n}(x)$ if and only if $b_{n-1}=b_{n}=0$ [3]. Thus, we must solve the nonlinear system

$$
b_{n-1}(p, q)=0, \quad b_{n}(p, q)=0 .
$$

Newton's method is used to accomplish this. The required partial derivatives are obtained recursively from

$$
c_{i}=b_{i}+p c_{i-1}+q c_{i-2}, \quad j=0, \cdots, n-1 ; c_{-2}=c_{-1}=0,
$$

where $\partial b_{n}(p, q) / \partial p=c_{n-1}, \partial b_{n}(p, q) / \partial q=\partial b_{n-1}(p, q) / \partial p=c_{n-2}$, and $\partial b_{n-1}(p, q) / \partial q=$ $c_{n-3}$.

The corrections to $p$ and $q$ are then

$$
\Delta p=\frac{b_{n} c_{n-3}-b_{n-1} c_{n-2}}{c_{n-2}^{2}-c_{n-1} c_{n-3}}, \quad \Delta q=\frac{b_{n-1} c_{n-1}-b_{n} c_{n-2}}{c_{n-2}^{2}-c_{n-1} c_{n-3}} .
$$

Thus, $p_{1}=p+\Delta p, q_{1}=q+\Delta q$ are the next approximations in this iterative process.

If $x^{2}-s x-t$ is a quadratic factor of $P_{n}(x)$, then the Jacobian determinant of (5) at the solution

$$
D(s, t)=\left|\begin{array}{cc}
\partial b_{n-1}(s, t) / \partial p & \partial b_{n-1}(s, t) / \partial q \\
\partial b_{n}(s, t) / \partial p & \partial b_{n}(s, t) / \partial q
\end{array}\right|
$$

is nonzero in case the quadratic factor has zeros which are either simple, distinct zeros of $P_{n}(x)$ [3], or real equal zeros of multiplicity two.* In such cases, Newton's method converges quadratically for sufficiently close initial guesses. In all other cases, $D(s, t)=$ $0, *$ thereby causing the method to converge slowly or to fail because of near zero divisors in (6).

4. Modified Bairstow's Method. We begin this section with an investigation of the higher order partial derivatives of (5). The recursion formulas of the Bairstow method are extended as follows.

Definition 1. Let $A_{i}^{0}=a_{i}, j=0, \cdots, n$, and define

$$
\begin{aligned}
A_{-2}^{i} & =A_{-1}^{i}=0, \\
A_{i}^{i} & =A_{i}^{i-1}+p A_{i-1}^{i}+q A_{i-2}^{i}, \quad j=0, \cdots, n ; \quad i=1,2, \cdots .
\end{aligned}
$$

* See Corollary 1 and Theorem 4 of the next section. 
We remark at this point that $A_{i}^{1} \equiv b_{i}$ and $A_{i}^{2} \equiv c_{i}$ by definition. $A_{i}^{i}$ is obviously a function of $p$ and $q$, and the point at which it is evaluated, if not explicitly stated, will be apparent from the context.

Definition 2. Given $P_{n}(x)$ and a particular $(p, q)$, let

$$
P_{n-2 k}(x)=A_{0}^{k} x^{n-2 k}+A_{1}^{k} x^{n-1-2 k}+\cdots+A_{n-2 k}^{k}, \quad k=0, \cdots,[n / 2] .
$$

As seen from (4), $P_{n-2}(x)$ is the quotient polynomial resulting from the division of $P_{n}(x)$ by $x^{2}-p x-q$. If $P_{n-2}(x)$ is divided by $x^{2}-p x-q$, the quotient would be $P_{n-4}(x)$. These results are contained in the following lemma.

LEMMA 1.

$$
\begin{aligned}
P_{n-2 k}(x)=\left(x^{2}-p x-q\right) P_{n-2-2 k}(x)+(x-p) A_{n-1-2 k}^{k+1}(p, q) & +A_{n-2 k}^{k+1}(p, q) \\
& \text { for } k=0, \cdots,[n / 2] .
\end{aligned}
$$

Proof. By induction on $k$. If $k=0$, we are considering the original Bairstow method, and this lemma has been established [3]. Assume the lemma true for $k-1$, and consider $k \leqq[n / 2]$. Then

$$
\begin{aligned}
P_{n-2 k}(x) & =A_{0}^{k} x^{n-2 k}+A_{1}^{k} x^{n-1-2 k}+\cdots+A_{n-2 k}^{k} \\
& =\left(x^{2}-p x-q\right) Q(x)+(x-p) d_{n-1-2 k}+d_{n-2 k}
\end{aligned}
$$

where

$$
Q(x)=d_{0} x^{n-2-2 k}+d_{1} x^{n-3-2 k}+\cdots+d_{n-2-2 k} .
$$

Equating coefficients of like powers of $x$ yields

$$
\begin{aligned}
& d_{0}=A_{0}^{k}, \\
& d_{1}=A_{1}^{k}+p d_{0}, \\
& d_{i}=A_{i}^{k}+p d_{i-1}+q d_{i-2}, \quad j=2, \cdots, n-2 k .
\end{aligned}
$$

From Definition 1 we see that $d_{i} \equiv A_{i}^{k+1}, j=0, \cdots, n-2 k$, and from Definition 2 that $Q(x) \equiv P_{n-2-2 k}(x)$, thereby establishing the lemma. Q.E.D.

We now proceed to show that the higher order partial derivatives of $b_{n}(p, q)$ and $b_{n-1}(p, q)$ may be obtained recursively from Definition 1 .

THEOREM 1.

$$
A_{i-1}^{i+1}=\frac{1}{i} \frac{\partial A_{i}^{i}}{\partial p}=\frac{1}{i} \frac{\partial A_{i+1}^{i}}{\partial q}, \quad j=0, \cdots, n ; i=1,2, \cdots .
$$

Proof. By induction on $i$. Suppose $i=1$ and $j=0$. Then $\partial A_{0}^{1} / \partial p=\partial A_{1}^{1} / \partial q=$ $A_{-1}^{2}=0$ is obvious. Assume the theorem is also true for subscripts $\leqq j$, and consider $j+1 \leqq n$. Then

$$
\begin{aligned}
\frac{\partial A_{i+1}^{1}}{\partial p} & =\frac{\partial}{\partial p}\left[A_{i+1}^{0}+p A_{i}^{1}+q A_{i-1}^{1}\right] \\
& =A_{i}^{1}+p A_{i-1}^{2}+q A_{i-2}^{2} \\
& =A_{i}^{2}
\end{aligned}
$$




$$
\begin{aligned}
\frac{\partial A_{j+2}^{1}}{\partial q} & =\frac{\partial}{\partial q}\left[A_{j+2}^{0}+p A_{i+1}^{1}+q A_{j}^{1}\right] \\
& =A_{i}^{1}+p A_{i-1}^{2}+q A_{j-2}^{2} \\
& =A_{i}^{2} .
\end{aligned}
$$

Thus, the theorem is true for $j+1$. Now assume it is true for $i$ and all $j, 0 \leqq j \leqq n$. Consider $i+1$. If $j=0$, then

$$
\frac{1}{i+1} \frac{\partial A_{0}^{i+1}}{\partial p}=\frac{1}{i+1} \frac{\partial A_{1}^{i+1}}{\partial q}=A_{-1}^{i+2}=0
$$

is readily apparent. Assume the theorem true for subscripts $\leqq j$, and consider $j+$ $1 \leqq n$. Then

$$
\begin{aligned}
\frac{1}{i+1} \frac{\partial A_{i+1}^{i+1}}{\partial p} & =\frac{1}{i+1} \frac{\partial}{\partial p}\left[A_{i+1}^{i}+p A_{j}^{i+1}+q A_{i-1}^{i+1}\right] \\
& =\frac{1}{i+1}\left[\frac{\partial A_{i+1}^{i}}{\partial p}+p \frac{\partial A_{i}^{i+1}}{\partial p}+A_{i}^{i+1}+q \frac{\partial A_{j-1}^{i+1}}{\partial p}\right] \\
& =\frac{1}{i+1}\left[i A_{j}^{i+1}+p(i+1) A_{j-1}^{i+2}+A_{i}^{i+1}+q(i+1) A_{i-2}^{i+2}\right] \\
& =A_{i}^{i+2}, \\
\frac{1}{i+1} \frac{\partial A_{j+2}^{i+1}}{\partial q} & =\frac{1}{i+1} \frac{\partial}{\partial q}\left[A_{j+2}^{i}+p A_{i+1}^{i+1}+q A_{j}^{i+1}\right] \\
& =\frac{1}{i+1}\left[\frac{\partial A_{j+2}^{i}}{\partial q}+p \frac{\partial A_{i+1}^{i+1}}{\partial q}+q \frac{\partial A_{j}^{i+1}}{\partial q}+A_{i}^{i+1}\right] \\
& =\frac{1}{i+1}\left[i A_{i}^{i+1}+p(i+1) A_{i-1}^{i+2}+q(i+1) A_{j-2}^{i+2}+A_{j}^{i+1}\right] \\
& =A_{i}^{i+2} .
\end{aligned}
$$

Thus, the theorem is true for $j+1$. Q.E.D.

THEOREM 2.

$$
\frac{\partial^{k} A_{j}^{1}}{\partial p^{k-i} \partial q^{i}}=k ! A_{i-i-k}^{k+1}, \quad j=0, \cdots, n ; \quad k=0, \cdots, j ; \quad i=0, \cdots, k .
$$

Proof. By induction on $k$. If $k=0$, the theorem is obvious. Assume the theorem true for $k$ and all $i, 0 \leqq i \leqq k$. Consider $k+1 \leqq j$. If $i=0$, then

$$
\frac{\partial^{k+1} A_{i}^{1}}{\partial p^{k+1}}=\frac{\partial}{\partial p}\left[\frac{\partial^{k} A_{i}^{1}}{\partial p^{k}}\right]=\frac{\partial}{\partial p}\left[k ! A_{i-k}^{k+1}\right]=(k+1) ! A_{i-k-1}^{k+2}
$$

Assume the theorem true for $i$, and consider $i+1 \leqq k+1$. Then

$$
\begin{aligned}
\frac{\partial^{k+1} A_{i}^{1}}{\partial p^{k+1-(i+1)} \partial q^{i+1}} & =\frac{\partial}{\partial q}\left[\frac{\partial^{k} A_{j}^{1}}{\partial p^{k-i} \partial q^{i}}\right]=\frac{\partial}{\partial q}\left[k ! A_{j-i-k}^{k+1}\right] \\
& =(k+1) ! A_{i-(k+1)-(i+1)}^{k+2} .
\end{aligned}
$$

Thus, the theorem is true for $i+1$. Q.E.D. 
The next theorem provides a criterion for the determination of the multiplicity of a quadratic factor.

THEOREM 3. $\left(x^{2}-s x-t\right)^{m}, m \geqq 1$, is a factor of $P_{n}(x)$ if and only if

$$
A_{n+1-2 k}^{k}(s, t)=A_{n+2-2 k}^{k}(s, t)=0 \quad \text { for } k=1, \cdots, m .
$$

Proof. By induction on $m$. If $m=1$, we have

$$
P_{n}(x)=\left(x^{2}-s x-t\right) P_{n-2}(x)+(x-s) A_{n-1}^{1}(s, t)+A_{n}^{1}(s, t) .
$$

The proof, in this case, is given by Henrici [3]. Now assume the theorem true for $m$, and consider $m+1$. We have, by the induction hypothesis,

$$
P_{n}(x)=\left(x^{2}-s x-t\right)^{m} P_{n-2 m}(x) .
$$

Consider

$$
P_{n-2 m}(x)=\left(x^{2}-s x-t\right) P_{n-2-2 m}(x)+(x-s) A_{n-1-2 m}^{m+1}(s, t)+A_{n-2 m}^{m+1}(s, t) .
$$

If $A_{n-1-2 m}^{m+1}=A_{n-2 m}^{m+1}=0$, then $x^{2}-s x-t$ is a factor of $P_{n-2 m}(x)$, and so $\left(x^{2}-s x-t\right)^{m+1}$ is a factor of $P_{n}(x)$. Conversely, suppose $\left(x^{2}-s x-t\right)^{m+1}$ is a factor of $P_{n}(x)$, and $x^{2}-s x-t=(x-\alpha)(x-\beta)$. Then

$$
\begin{aligned}
& P_{n-2 m}(\alpha)=(\alpha-s) A_{n-1-2 m}^{m+1}+A_{n-2 m}^{m+1}=0, \\
& P_{n-2 m}(\beta)=(\beta-s) A_{n-1-2 m}^{m+1}+A_{n-2 m}^{m+1}=0 .
\end{aligned}
$$

If $\alpha \neq \beta$, the determinant of the system (8) is $\alpha-\beta \neq 0$, so the only solution of (8) is the trivial one $A_{n-1-2 m}^{m+1}=A_{n-2 m}^{m+1}=0$. However, if $\alpha-\beta=0$, then $P_{n-2 m}^{\prime}(\alpha)=$ $A_{n-1-2 m}^{m+1}=0$, implying that $A_{n-2 m}^{m+1}=0$ also. Q.E.D.

We propose to replace the system (5) of Bairstow's method by

$$
A_{n+1-2 m}^{m}(p, q)=0, \quad A_{m+2-2 m}^{m}(p, q)=0 .
$$

From Theorem 3 it is seen that $(s, t)$ is a solution of (9). To investigate the applicability of Newton's method to (9), its Jacobian determinant must be considered. To this end, we make the following definition.

Definition 3.

$$
J_{k}(p, q)=(k-1)^{2}\left|\begin{array}{ll}
A_{n+2-2 k}^{k}(p, q) & A_{n+3-2 k}^{k}(p, q) \\
A_{n+1-2 k}^{k}(p, q) & A_{n+2-2 k}^{k}(p, q)
\end{array}\right| \text { for } k=2,3, \cdots
$$

Using Theorem 1 it is seen that $J_{k+1}(p, q)$ is the Jacobian determinant of

$$
A_{n+1-2 k}^{k}(p, q)=0, \quad A_{n+2-2 k}^{k}(p, q)=0,
$$

and in particular, $J_{m+1}(p, q)$ is the Jacobian determinant of (9). (Note that $D(p, q)=$ $J_{2}(p, q)$.) An immediate consequence of Theorem 3 is

COROLlaRY 1. If $\left(x^{2}-s x-t\right)^{m}, m \geqq 2$, is a factor of $P_{n}(x)$, then

$$
J_{k+1}(s, t)=0 \quad \text { for } k=1, \cdots, m-1 .
$$

The following theorem establishes when $J_{m+1}(p, q)$ is nonzero.

THEOREM 4. Assume

(i) $\left(x^{2}-s x-t\right)^{m}$ is a factor of $P_{n}(x)$,

(ii) $\left(x^{2}-s x-t\right)^{m+1}$ is not a factor of $P_{n}(x)$, 
(iii) $x^{2}-s x-t=(x-\alpha)(x-\beta)$.

Then $J_{m+1}(s, t) \neq 0$ if and only if $P_{n-2 m}(\alpha) \neq 0$ and $P_{n-2 m}(\beta) \neq 0$.

Proof.

$$
\begin{aligned}
\frac{1}{m^{2}}\left[J_{m+1}(s, t)\right] & =\left[A_{n-2 m}^{m+1}(s, t)\right]^{2}-A_{n+1-2 m}^{m+1}(s, t) \cdot A_{n-1-2 m}^{m+1}(s, t) \\
& =\left[A_{n-2 m}^{m+1}\right]^{2}-\left[A_{n+1-2 m}^{m}+s A_{n-2 m}^{m+1}+t A_{n-1-2 m}^{m+1}\right] \cdot A_{n-1-2 m}^{m+1} \\
& =\left[A_{n-2 m}^{m+1}\right]^{2}-s A_{n-2 m}^{m+1} \cdot A_{n-1-2 m}^{m+1}-t\left[A_{n-1-2 m}^{m+1}\right]^{2} \\
& =\left[A_{n-2 m}^{m+1}\right]^{2}-(\alpha+\beta) A_{n-2 m}^{m+1} \cdot A_{n-1-2 m}^{m+1}+\alpha \beta \cdot\left[A_{n-1-2 m}^{m+1}\right]^{2} \\
& =\left[(\alpha-s) A_{n-1-2 m}^{m+1}+A_{n-2 m}^{m+1}\right] \cdot\left[(\beta-s) A_{n-1-2 m}^{m+1}+A_{n-2 m}^{m+1}\right] \\
& =P_{n-2 m}(\alpha) \cdot P_{n-2 m}(\beta) .
\end{aligned}
$$

The conclusion now follows immediately. Q.E.D.

As an immediate consequence of this theorem, we have the following corollary.

Corollary 2. Under assumptions (i), (ii) and (iii) of Theorem $4, J_{m+1}(s, t)=0$ if and only if $\alpha$ and $\beta$ are real and one of the following conditions occurs:

(a) $\alpha=\beta$ and $\alpha$ is of odd multiplicity.

(b) $\alpha \neq \beta$ and $m=$ multiplicity of $\alpha<$ multiplicity of $\beta$.

Newton's method, when applied to (9), converges quadratically for sufficiently close initial guesses provided its Jacobian determinant at the solution is nonzero. The corrections, $\Delta p$ and $\Delta q$, are given by

$$
\begin{aligned}
& \Delta p=\frac{m}{J_{m+1}(p, q)}\left[A_{n+2-2 m}^{m} \cdot A_{n-1-2 m}^{m+1}-A_{n+1-2 m}^{m} \cdot A_{n-2 m}^{m+1}\right], \\
& \Delta q=\frac{m}{J_{m+1}(p, q)}\left[A_{n+1-2 m}^{m} \cdot A_{n+1-2 m}^{m+1}-A_{n+2-2 m}^{m} \cdot A_{n-2 m}^{m+1}\right],
\end{aligned}
$$

where the $A_{i}^{i}$ are evaluated at $(p, q)$.

Under the conditions of Theorem 4, if we knew the value of $m$ we could solve (9) remembering that a solution of (5) is a solution of (9), but not conversely. Since in practice $m$ is not known in advance, we use an approximation to $m$ which is improved as the iteration continues. The following steps are suggested:

1. Given an initial guess, $\left(p_{0}, q_{0}\right)$, evaluate the $A_{i}^{i}$.

2. Estimate the value of $m$ by considering Theorem 3 .

3. Using this estimate in (11), calculate $\Delta p$ and $\Delta q$, and form the next iterate $p=p_{0}+\Delta p, q=q_{0}+\Delta q$.

4. Repeat the above using $(p, q)$ as a new initial guess.

In effect we solve system (10) at first with $k<m$, and finally with $k=m$ (i.e., system (9)). When $k<m$, system (10) has a zero Jacobian determinant at the solution $(s, t)$ by Corollary 1 . In this case, Newton's method is not quadratically convergent. In practice, however, only a few iterations per system need be taken before (9) is considered.

5. Results. The following criterion to determine $m$ has been used with success: $\mu$ is taken as an approximation to $m$ in case 


$$
\left|A_{n+1-2 k}^{k}\right| \leqq\left|a_{n}\right| \cdot \epsilon, \quad \text { and } \quad\left|A_{n+2-2 k}^{k}\right| \leqq\left|a_{n}\right| \cdot \epsilon
$$

for $k=1, \cdots, \mu .\left(\epsilon=10^{-4}\right.$ is a reasonable choice. $)$

This criterion in conjunction with the aforementioned root-finding algorithm was tested with 100 arbitrarily selected polynomials of degrees between 4 and 24 having multiple quadratic factors. This provided 175 test cases involving quadratic factors with multiplicity. Convergence was achieved in 103 instances $(59 \%)$ (see Table 1a). Divergence was noted only after seven attempts with various initial guesses, $\left(p_{0}, q_{0}\right)$, failed to produce a sequence which approached the proper solution. Excluding divergent cases, convergence was achieved $76 \%$ of the time. Bairstow's method was far inferior to the modified method. Typical results are shown in Table 2.

Clearly, the choice of criterion to determine multiplicity affects the overall performance of the algorithm since, as noted previously, convergence is less than quadratic when $k<m$. It would appear, therefore, that the correct value of the multiplicity should be used as soon as possible. Experience has shown, however, that the regions of convergence differ when $m$ is known as compared to when $m$ is calculated using (12). Closer initial guesses may be required when $m$ is known.

For example, consider the results for the polynomial $\left(x^{2}+9\right)^{3}(x-3)^{6}(x-2)^{2}$ given in Table 3. For case (b) when $m$ is known, convergence is to an extraneous solution. A closer initial guess is required to achieve convergence to the desired quadratic (case (d)). Case (a) shows that by using a sequence of approximations to $m$ in lieu of a closer guess, the desired convergence may also be obtained. Usually when $m$ is calculated, more iterations are necessary to obtain convergence than when $m$ is known (cases (c) and (d)). (Similar results were observed for Rall's method which is described in Section 6.)

6. The Relationship to Rall's Method. Using the notation of Section 3, Rall's method [4] provides corrections

$$
\Delta p=m \frac{b_{n} c_{n-3}-b_{n-1} c_{n-2}}{c_{n-2}^{2}-c_{n-1} c_{n-3}}, \quad \Delta q=m \frac{b_{n-1} c_{n-1}-b_{n} c_{n-2}}{c_{n-2}^{2}-c_{n-1} c_{n-3}}
$$

From Theorem 2 we see that (11) involves $(m-1)$ st and $m$ th order partial derivatives of $b_{n}$ and $b_{n-1}$, whereas (6) and (13) involve only $b_{n}, b_{n-1}$ and their first-order partials. Clearly, using the recursion of Definition 1, (11) costs more to compute than (13). Analogous to Section 2, however, we may have to calculate higher order partial derivatives anyway in order to determine $m$ using Theorem 3. If such were the case, suppose we have determined that $A_{n+1-2 k}^{k}$ and $A_{n+2-2 k}^{k}$ are negligible for $k=1, \cdots, m$, but are not negligible when $k=m+1$ (i.e., $A_{n-1-2 m}^{m+1}$ and $A_{n-2 m}^{m+1}$ are not negligible). The quantities $A_{n+1-2 m}^{m}, A_{n+2-2 m}^{m}, A_{n-1-2 m}^{m+1}, A_{n-2 m}^{m+1}$, and $A_{n+1-2 m}^{m+1}$ are required to form $\Delta p$ and $\Delta q$ using (11). All but the last of these have been computed. The quantities required by (13) are available so the extra cost in using (11) instead of (13) is the computation of $A_{n+1-2 m}^{m+1}$. Both (11) and (13) are quadratically convergent, so it would appear that (13) is preferable in light of its lesser cost. We claim, however, that numerical difficulties are possible as the denominator in (13) approaches zero.

The method was tested with the previously mentioned data set both with $m$ assumed and with $m$ calculated using (12) (see Table $1 b$ ). In the former case, convergence occurred in only three instances. Failure due to small (less than or equal to $\delta=0.5$ $\times 10^{-8}$ in magnitude) divisors in (13) occurred in 109 cases $(62 \%)$. In such instances 


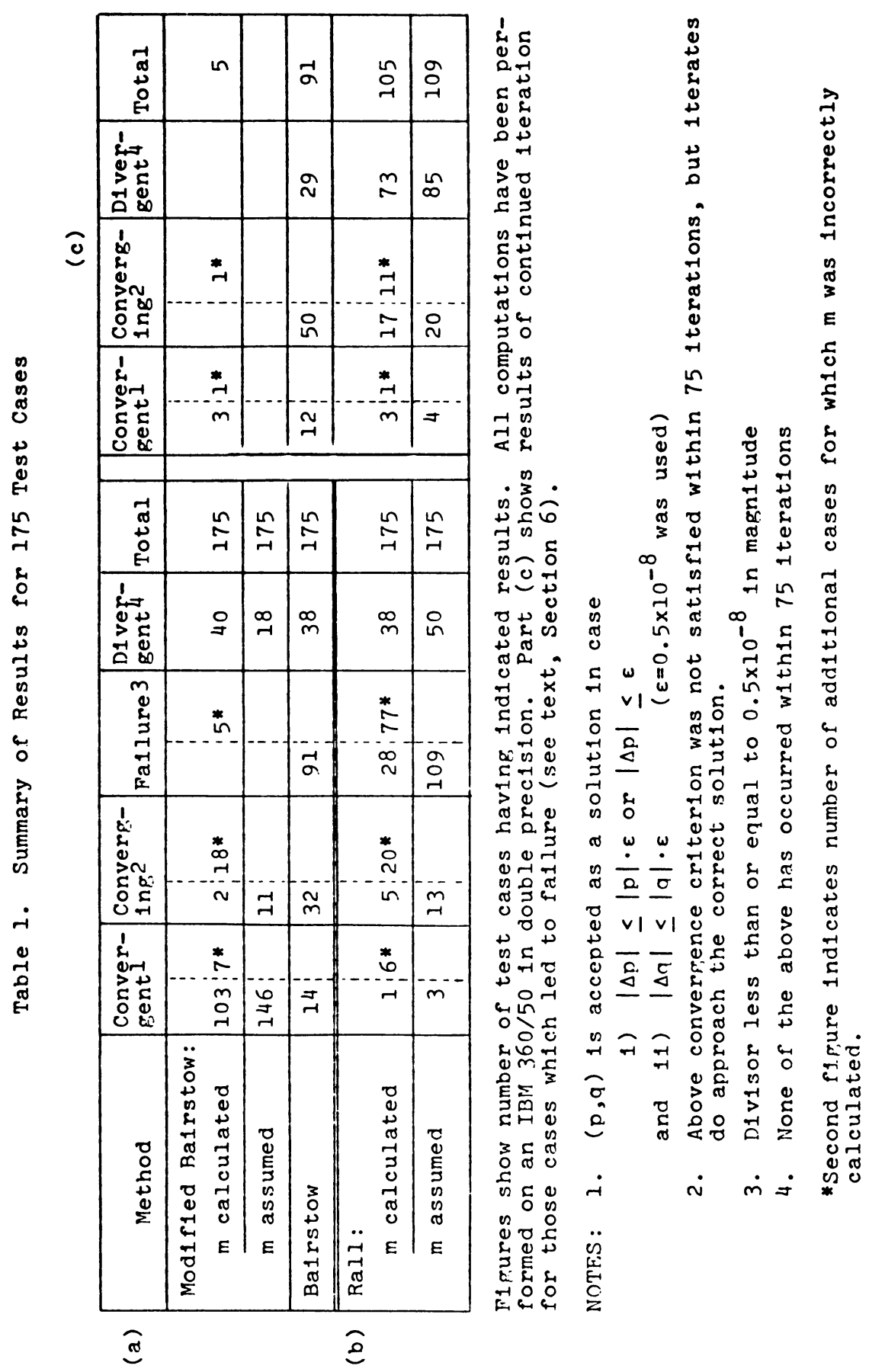




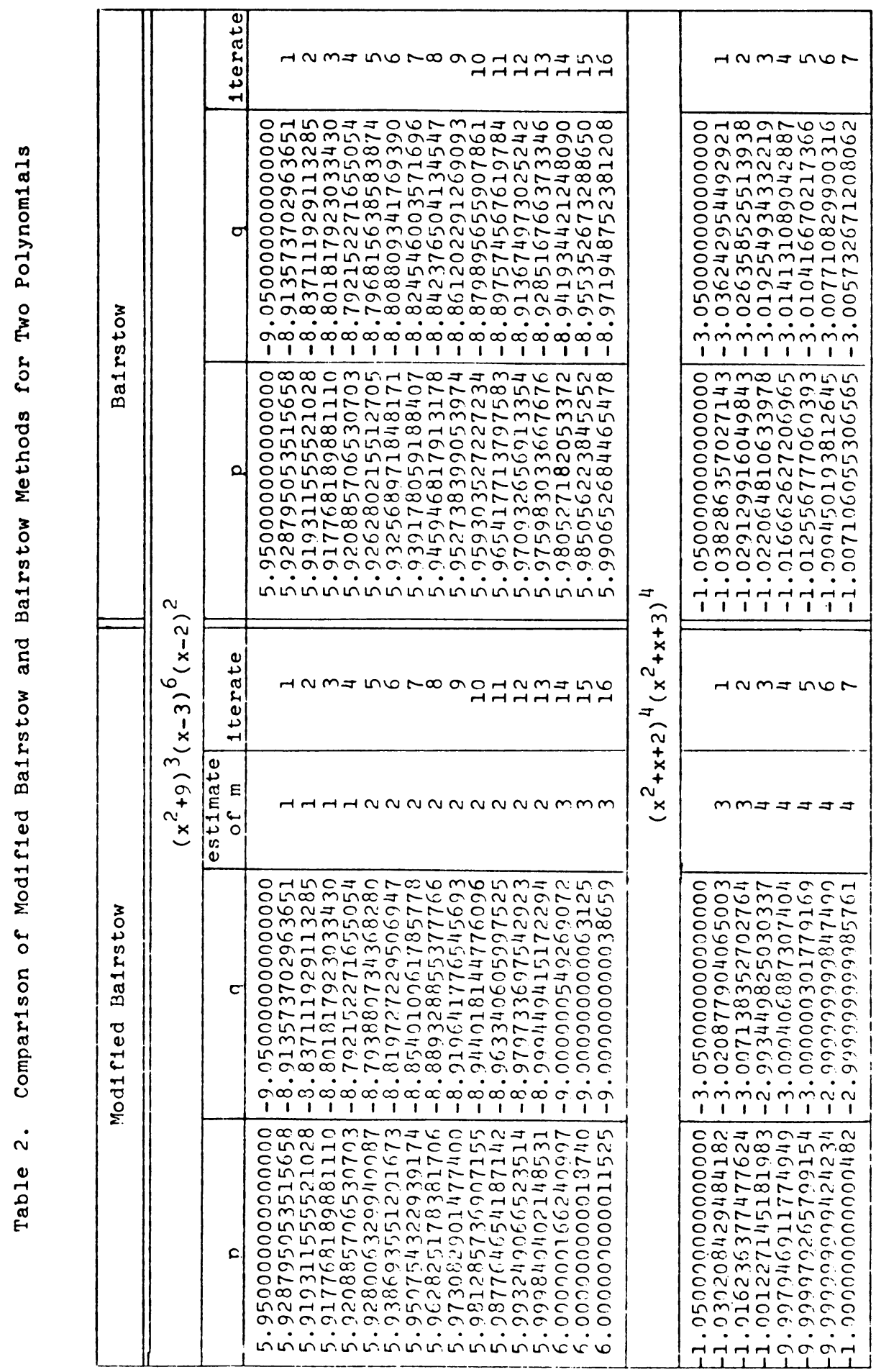




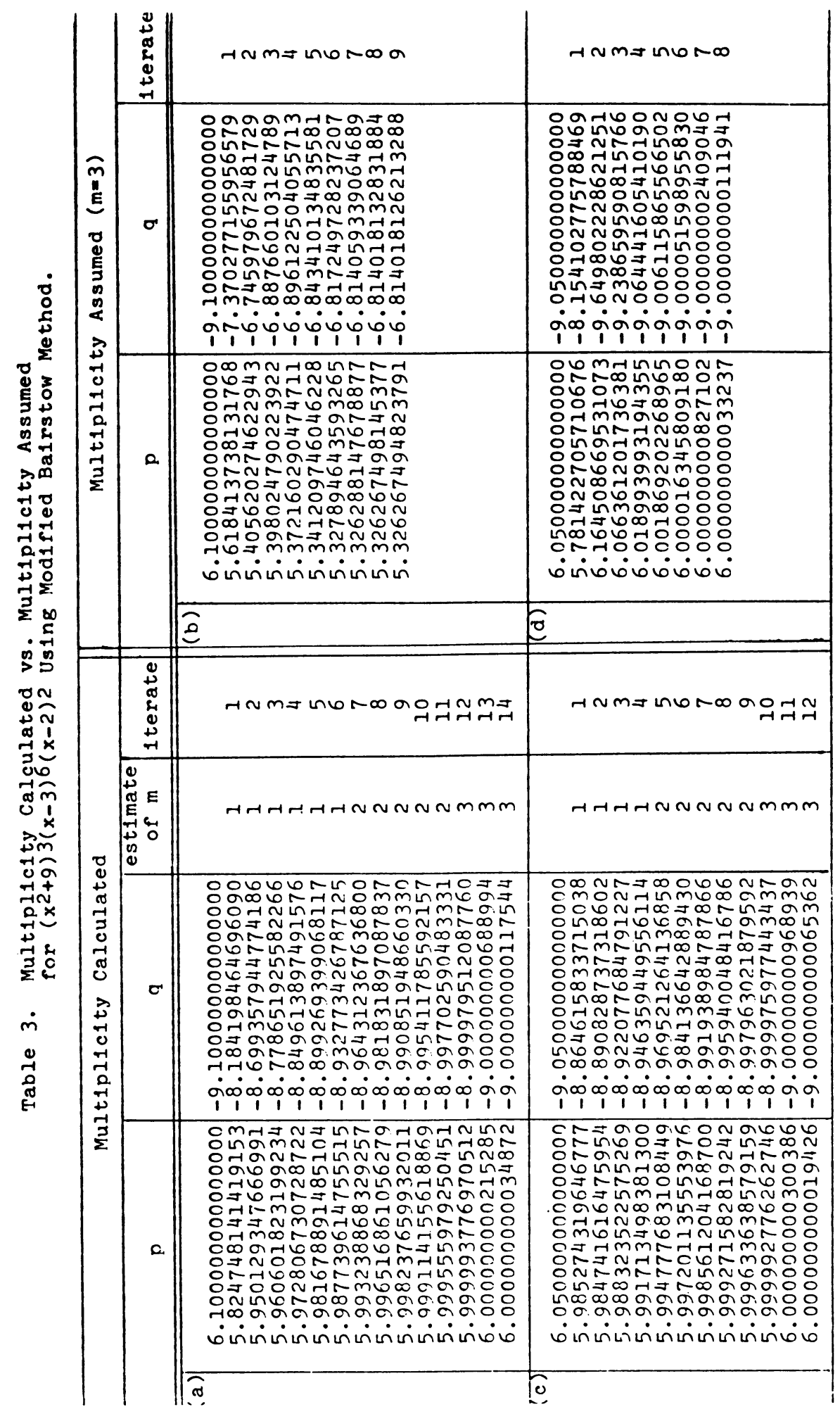




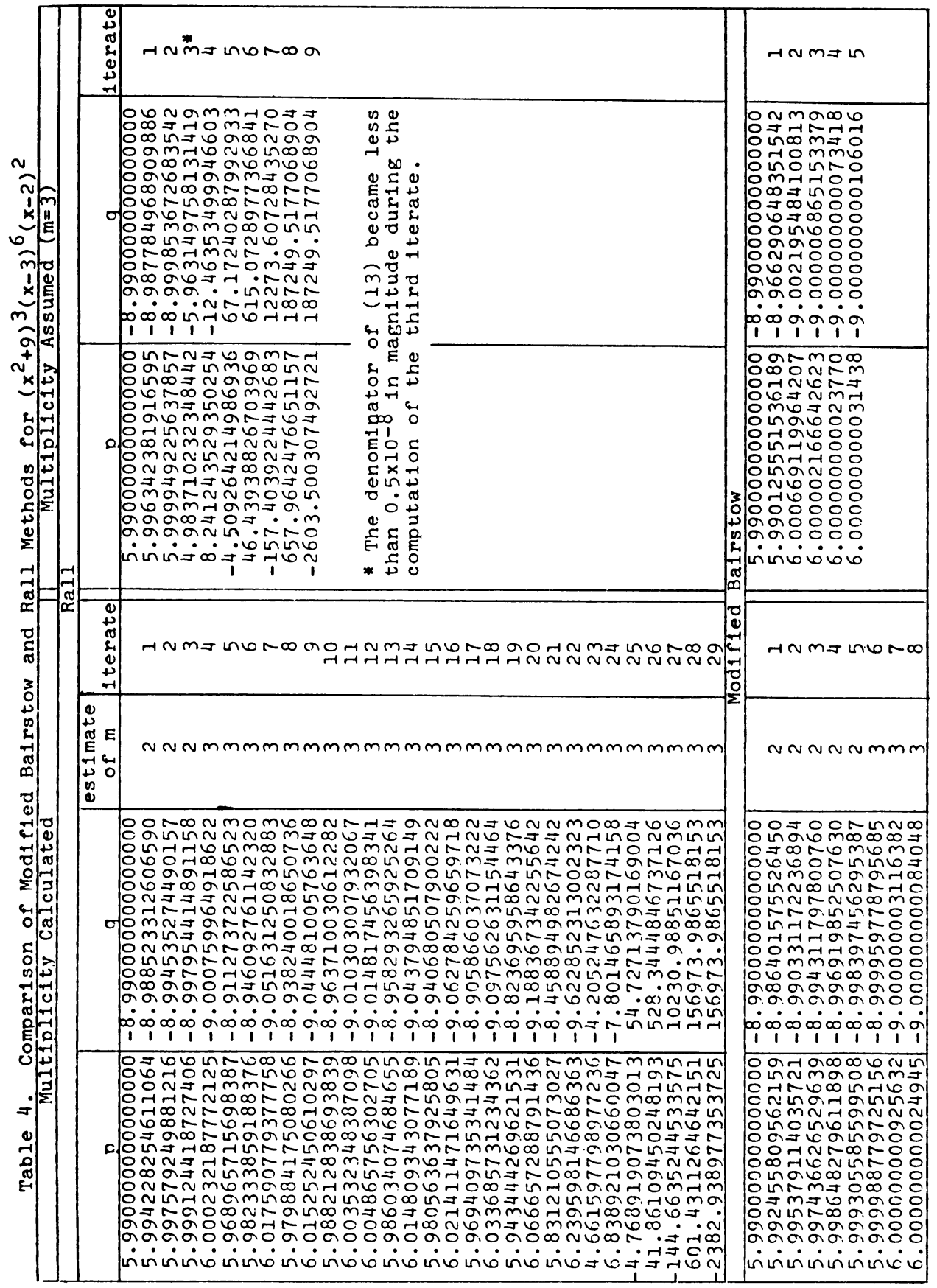


iteration was resumed to insure that the choice of $\delta$ did not bias the results. Failure was recognized only at the occurrence of an exponent underflow or overflow (this, in fact, never took place). Of the 109 cases, 85 resulted in divergence, 20 involved sequences which approached the solution very slowly, while for the remaining 4 convergence actually took place (see Table $1 \mathrm{c}$ ). Thus, continued iteration did not prove to be profitable. Results were slightly better when $m$ was computed, but the overall behavior was essentially the same.

Typical results are shown in Table 4. Here Rall's method with $m$ assumed begins to converge. The denominator of (13) is less than $0.5 \times 10^{-8}$ in magnitude when the third iterate is computed. Subsequently, the sequences diverge. For comparison purposes we consider the modified Bairstow method under the same conditions. Convergence is obtained after five iterations. We note, however, that the second iterate is less accurate than the second iterate of Rall's method ((6.00067, -9.00220) vs. (5.99995, -8.99985)). Nevertheless, greater accuracy is ultimately achieved using the modified Bairstow method. Similar results are obtained when $m$ is calculated using (12). Note, however, that the denominator of (13) does not become less than $0.5 \times 10^{-8}$ in magnitude.

Acknowledgement. The author wishes to thank Professor Paul W. Gilbert and Mrs. Phyllis Kent of Syracuse University for inspiring this work.

Department of Computer Science and Experimental Statistics

University of Rhode Island

Kingston, Rhode Island 02881

1. L. BaIrstow, Investigations Relating to the Stability of the Aeroplane, Reports and Memoranda \#154, Advisory Committee for Aeronautics, October 1914, pp. 51-64.

2. F. M. Carrano, A Generalized Bairstow Method for Multiple Zeros of a Polynomial, Ph.D. Dissertation, Syracuse University, Syracuse, N.Y., 1969.

3. P. HENRICI, Elements of Numerical Analysis, Wiley, New York, 1964. MR 29 \#173.

4. L. B. Rall, "Convergence of the Newton process to multiple solutions," Numer. Math., v. 9, 1966, pp. 23-37. MR 35 \#1209. 